\title{
A dual origin for Neptune's carbon monoxide?
}

\author{
E. Lellouch ${ }^{1}$, R. Moreno ${ }^{1}$, and G. Paubert ${ }^{2}$ \\ ${ }^{1}$ Laboratoire d'Études Spatiales et d'Instrumentation en Astrophysique (LESIA), Observatoire de Paris, 92195 Meudon, \\ France \\ e-mail: emmanuel.lellouch@obspm.fr \\ 2 Institut de Radio-Astronomie Millimétrique, 18080 Granada, Spain
}

Received 5 November 2004 / Accepted 14 December 2004

\begin{abstract}
Heterodyne observations of Neptune have provided a measurement of the $\mathrm{CO}(2-1)$ line profile with a total bandpass of almost $8 \mathrm{GHz}$ and a resolution of $4 \mathrm{MHz}$. The lineshape indicates that the $\mathrm{CO}$ mole fraction in Neptune's atmosphere is not uniform, but increases by a factor of $\sim 2$ from the troposphere/lower stratosphere $(0.5 \mathrm{ppm}$ at $p>20 \mathrm{mbar})$ to the upper stratosphere ( $1 \mathrm{ppm}$ at $p<20 \mathrm{mbar}$ ). This indicates the existence of both external and internal sources of CO. The equivalent flux associated with the external source is $\sim 1 \times 10^{8} \mathrm{~cm}^{-2} \mathrm{~s}^{-1}$. We propose that the stratospheric CO results from a large $(2 \mathrm{~km})$ cometary impact that occurred $\sim 200$ years ago, although there remains problems with this hypothesis.
\end{abstract}

Key words. planets and satellites: Neptune - radio-lines: solar system

\section{Introduction}

Though they are "twins" in terms of their main properties (radius, mass, density, bulk composition), Uranus and Neptune present drastic differences as to their internal structure, cloud activity, and detailed composition. While Neptune emits about $60 \%$ more energy than it receives from the Sun, implying the presence of a strong internal energy source, Uranus lacks such an internal source. This difference is thought to be responsible for the weaker dynamical and meteorological activity in Uranus. It is also probably at the origin of differences in the chemical composition at observable levels (at $p \leq 1$ bar). In particular, the presence of carbon monoxide in Neptune's stratosphere in large amounts $\left((0.6-1) \times 10^{-6}\right.$; Marten et al. 1993; Rosenqvist et al. 1992), established more than a decade ago from millimeter observations, is generally interpreted as resulting from vigorous upward convection from the deep interior where $\mathrm{CO}$ is stable. Concommitant observations failed to detect $\mathrm{CO}$ on Uranus, with an upper limit of $1 / 15$ of the Neptune value (Marten et al. 1993). This absence is interpreted as due to a much more sluggish vertical transport in the case of Uranus.

The case for Neptune's CO being of internal origin is primarily made from the claim (Marten et al. 1993; Guilloteau et al. 1993; Naylor et al. 1994) that the CO stratospheric mixing ratio also reflects its abundance in Neptune's troposphere. However, for this, the first two papers used broad-band measurements in the vicinity of the $\mathrm{CO}(3-2)$ and (1-0) lines. Naylor et al. (1994) reported a clear detection of the $\mathrm{CO}(3-$ 2) line in absorption from FTS/JCMT observations, but the quality of their spectra was limited by channel fringing. From similar measurements in the $\mathrm{CO}(2-1)$ line, Encrenaz et al. (1996) rather inferred a 3- $\sigma$ upper limit of $1 \mathrm{ppm}$ for CO in Neptune's troposphere. Thus none of the earlier observations have allowed a satisfactory determination of the CO line profiles; in addition, the presence of an absorption feature does not per se imply the presence of CO in Neptune's troposphere.

From the theoretical standpoint, models that attempt to explain a $\mathrm{CO}$ abundance of $1 \mathrm{ppm}$ in the bulk of Neptune's atmosphere have to invoke an oxygen enhancement in Neptune's interior $\mathrm{O} / \mathrm{H}=440$ times solar (Lodders \& Fegley 1994), an extraordinarily high value that implies that water constitutes $60 \%$ of the gas phase in Neptune's interior.

The last decade has brought a wealth of observations that have shed new light on the origin of oxygen compounds in Outer Planets. First, the Shoemaker-Levy 9 collision with Jupiter has revealed that cometary impacts may supply large amounts of $\mathrm{CO}$ and $\mathrm{H}_{2} \mathrm{O}$ to planetary atmospheres (see e.g. review in Lellouch 1995). The discovery by ISO of $\mathrm{H}_{2} \mathrm{O}$ and $\mathrm{CO}_{2}$ in the atmospheres of the Giant Planets and Titan (Feuchtgruber et al. 1997; Coustenis et al. 1998), has provided a second proof for an external supply of oxygen to the Outer Planets in the form or water and probably additional species ( $\mathrm{CO}, \mathrm{CO}_{2}, \mathrm{CH}_{3} \mathrm{OH}$..., see Moses et al. 2000), and the likely role of interplanetary micrometeorites, planetary environments (satellites, rings) and cometary impacts has been demonstrated (Lellouch et al. 2002). In Jupiter's case, the existence of two distinct sources of carbon monoxide (besides the recent SL9 event), resulting respectively from internal transport and (probably) episodic cometary deposition, has been clearly established (Bézard et al. 2002). Finally, CO was recently detected in Uranus with a mixing ratio of $\sim 3 \times 10^{-8}$ at $0.1-1$ bar, 
Table 1. Observational details.

\begin{tabular}{ccc}
\hline \hline Setup & $\begin{array}{c}\text { Central } \\
\text { frequencies }(\mathrm{GHz})\end{array}$ & Date(s) \\
\hline 1 & $230.154,230.922$ & $08 / 15 / 03,05 / 12 / 04,05 / 13 / 04$ \\
2 & $228.618,229.386$ & $08 / 15 / 03,05 / 12 / 04$ \\
3 & $231.690,232.458$ & $09 / 01 / 03,05 / 13 / 04$ \\
4 & $227.082,227.850$ & $05 / 12 / 04$ \\
5 & $233.226,233.994$ & $05 / 13 / 04$ \\
\hline
\end{tabular}

and an external origin was tentatively favored (Encrenaz et al. 2004).

In this context, it appears necessary to reassess the origin of carbon monoxide on Neptune. Here, we present new observations of the $\mathrm{CO}(2-1)$ line, that provide a better characterization of its absorption component and from which the vertical distribution of $\mathrm{CO}$ is inferred.

\section{Observations and data reduction}

Neptune observations were acquired with the IRAM 30-m telescope during the nights of August 15, 2003, September 1, 2003, May 12, 2004 and May 13, 2004. The basic idea of the experiment was to achieve an optimum determination of the CO (2-1) $230.538 \mathrm{GHz}$ line shape over the largest possible frequency interval. We used two $1.3 \mathrm{~mm}$ receivers in parallel, tuning each of them at a frequency close to the CO line (this defines one "setup"). For spectral analysis, we used two backends covering $1024 \mathrm{MHz}$ each at a resolution of $4 \mathrm{MHz}$, i.e. $\sim 2 \mathrm{GHz}$ were covered instantaneously. The strategy consisted of acquiring relatively short integrations (typically $1 / 2 \mathrm{~h}$ ) on each setup, and then move to another setup. To facilitate the definition of the global line shape, we imposed overlaps of the individual bands, by a quarter of their bandwidths (i.e. $250 \mathrm{MHz}$ ). This allowed, in the data reduction phase, the individual pieces of $1 \mathrm{GHz}$ to be joined smoothly. In contrast, we did not make any special effort to achieve an optimum absolute calibration. Table 1 summarizes the various setups that were used on the four observing dates. In total, the CO line was covered over a $7.936 \mathrm{GHz}$ range.

Figure 1 shows all observations, in antenna temperature scale. Due to calibration uncertainties, pointing uncertainties, and residual sky fluctuations, the individual pieces do not, in general, line up "naturally". A notable exception is provided by the August 15, observations, which cover the 226.572-231.432 GHz range, and clearly show the CO absorption without any need for piecewise adjustment. The observations of August 15, May 12 and May 13 encompass the $\mathrm{CO}$ emission core. After calibration, the measured antenna temperature minimum at the foot of the emission $(230.470 \mathrm{GHz})$ is $56.1,47.5$ and $64.4 \mathrm{~K}$ for the three observations respectively. Radiative transfer models predict a value of $64 \mathrm{~K}$ at this frequency for a uniform $\mathrm{CO}=1 \times 10^{-6}$ mixing ratio. Allowing for a $15 \%$ calibration uncertainty, the May 12 observations thus show a significant flux deficit compared to expectations. We therefore treat these observations with caution, but given that they detect the $\mathrm{CO}$ emission with appropriate line-to-continuum contrast, we do not discard them. The

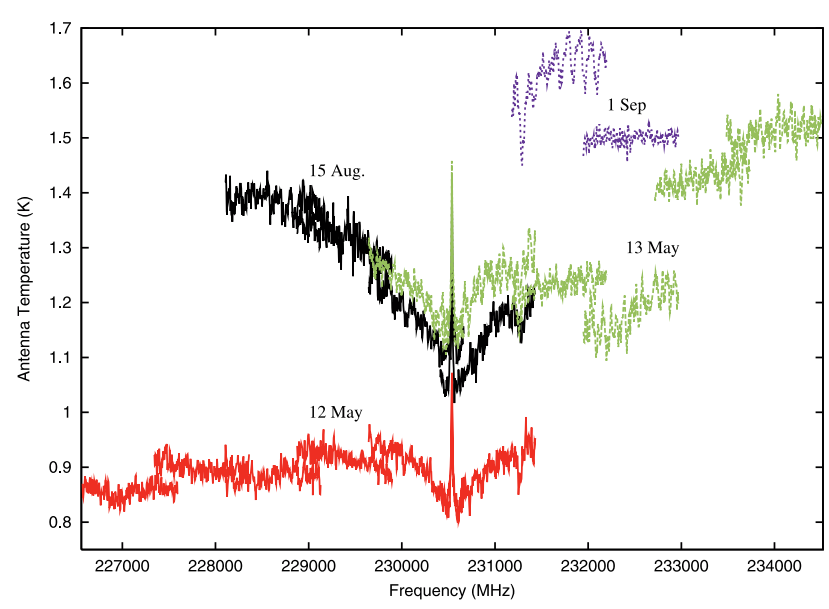

Fig. 1. An overview of all observations. Data are plotted directly in antenna temperatures, i.e. are not corrected for forward and main beam efficiencies, or Neptune's filling factor. Black: Aug. 15, 2003. Blue (dashed line): Sep. 1, 2004. Red: May 12, 2004. Green (dashed line): May 13, 2004. The absorption near $231280 \mathrm{MHz}$ is due to telluric ozone.

procedure to restore the full shape of the $\mathrm{CO}$ line was as follows. All individual pieces were first averaged according to their central frequency. Then, the two pieces corresponding to setup 1 were rescaled individually by a constant factor so that their common part $(230.412-230.664 \mathrm{GHz})$ matches the averaged value in this range (measured 6 times). Pieces corresponding to other setups were then rescaled sequentially, to ensure proper overlap with adjacent bands. The resulting line shape is shown in Figs. 2 and 3, in which the precise vertical scale is unimportant. Our procedure thus applies multiplicative factors to the various pieces of the spectrum, rather than shifting them vertically, as would be appropriate if (additive) sky fluctuations were the dominant cause of flux dispersion. Justification is provided by the fact that the constrast of the $\mathrm{CO}$ emission scales with the local continuum rather than being constant from one day to another. We note, anyway, that given the restricted frequency coverage of the individual pieces, applying vertical shifts would produce very small changes in the CO profile. The final CO line profile shows asymmetry in the far-wings ( $>2 \mathrm{GHz}$ from line center), and given the caveats above on the May 12 observations, we regard the high-frequency wing as the most reliable.

\section{Modelling}

The obtained CO line shape was modelled using a standard radiative transfer model (Moreno 1998) of millimeter spectra of the Giant Planets. Opacity due to $\mathrm{CO}, \mathrm{NH}_{3}$ far wings, and the $\mathrm{H}_{2}-\mathrm{He}-\mathrm{CH}_{4}$ pressure-induced continuum were included. The Neptune thermal profile, as well as standard parameters (e.g. $\mathrm{He} / \mathrm{H}_{2}$ ratio), were taken from Bézard et al. (1991). Models were convolved to the instrumental $4 \mathrm{MHz}$ resolution. Neptune's rapid rotation ( $2.7 \mathrm{~km} \mathrm{~s}^{-1}$ at equator) was included and found to have a small effect. Given the uncertain temperature scale of our data, only constraints derived from modelling the line shape were considered. For this, all models were 

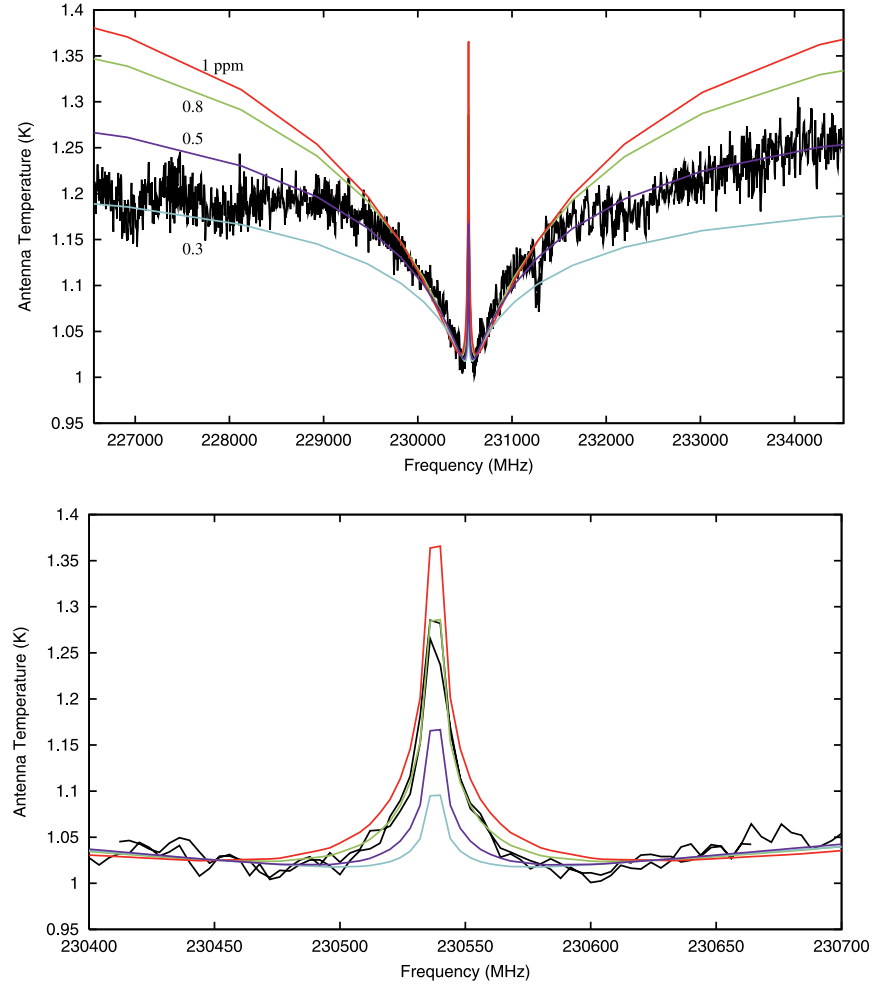

Fig. 2. Models of the total CO line with uniform $\mathrm{CO}$ mole fraction. Data: solid black lines. Models (from top to bottom): red: $\mathrm{CO}=1.0 \mathrm{ppm}$; green: $0.8 \mathrm{ppm}$; dark blue: $0.5 \mathrm{ppm}$; light blue: $0.3 \mathrm{ppm}$.

multiplied by constant factors to match the data in the region of maximum absorption near $230.450 \mathrm{GHz}$.

Figure 2 shows a comparison of the data with models in which $\mathrm{CO}$ is assumed to be well mixed throughout Neptune's atmosphere, with mole fractions ranging from 0.3 to $1.0 \mathrm{ppm}$. A value of $\sim 0.8 \mathrm{ppm}$ matches the central emission, consistent with the initial findings of Marten et al. (1993) and Rosenqvist et al. (1992). However, while abundances of 0.8-1 ppm also provide a satisfactory match of the absorption up to $\sim 1.5 \mathrm{GHz}$ from line center, they do not allow to fit the more distant wings, which are more flat, and rather suggest a mole fraction of $0.5 \pm 0.1 \mathrm{ppm}$. The same conclusion is reached even if only the best quality August 15 observations, which extend up to $2.4 \mathrm{GHz}$ from line center, are considered. We tested its robustness to temperature uncertainties by using an alternate thermal profile, based on the results of Bézard et al. (1999) at $p>10$ mbar, Marten et al. (2005) at $p<1 \mathrm{mbar}$, and interpolation between. In the troposphere, this profile is warmer than ours by $\sim 2 \mathrm{~K}$ at 2 bar and $\sim 4 \mathrm{~K}$ at 0.1 bar. With this profile, the far wings and central emission are fit respectively with $\mathrm{CO}$ abundances of 0.65 and $0.9 \mathrm{ppm}$, however, the close wings $(0.2-0.8 \mathrm{GHz}$ from line center) are significantly too broad. We conclude that the data indicate a non-uniformity of the CO mixing ratio in Neptune's stratosphere, and proceed to test vertically-varying models.

The presence of $\mathrm{CO}$ in Neptune's stratosphere is directly demonstrated by the existence of the emission core. In contrast, the absorption feature does not, in itself, prove that $\mathrm{CO}$
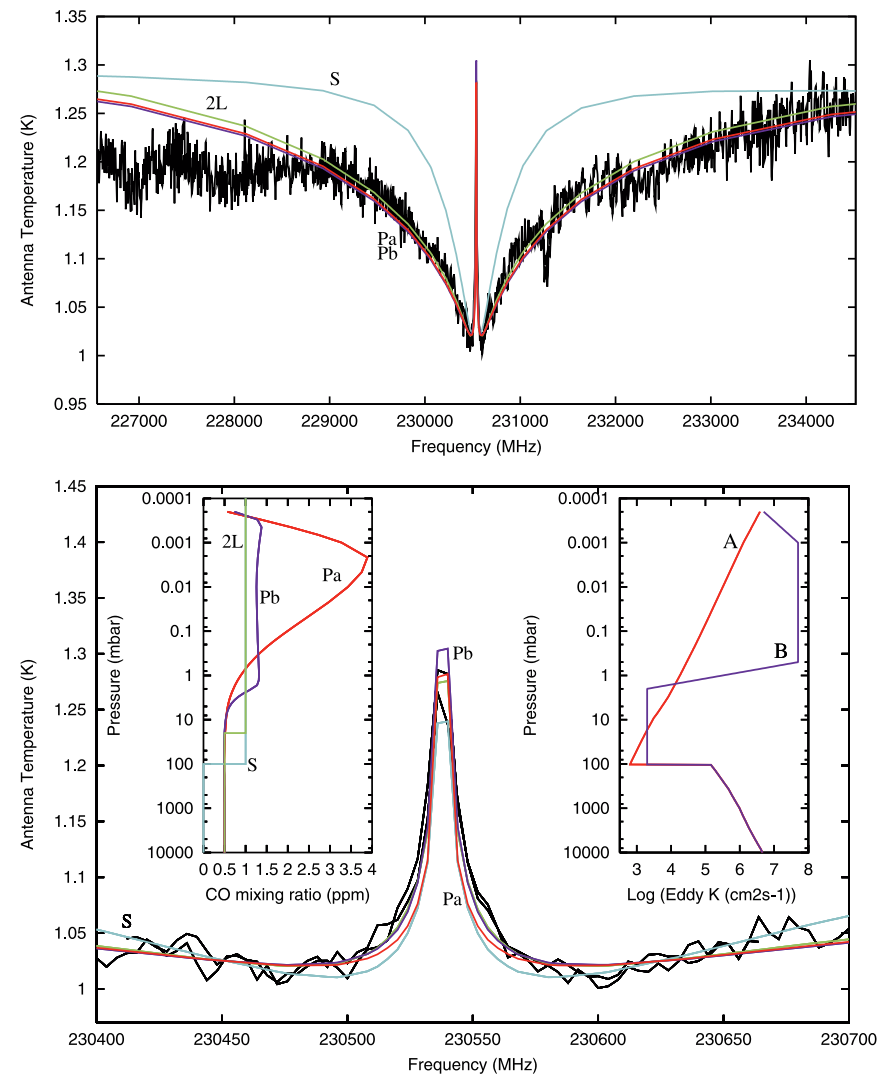

Fig. 3. Models with altitude-varying $\mathrm{CO}$ mixing profiles. Light blue (label S): CO restricted to $p<100$ mbar with $1.0 \mathrm{ppm}$ mole fraction. Green (label 2L): two-level model, with $\mathrm{CO}=1.0 \mathrm{ppm}$ at $p<$ 20 mbar and $\mathrm{CO}=0.5 \mathrm{ppm}$ at $p>20 \mathrm{mbar}$. Red (label Pa): physical model, with $\mathrm{CO}=0.5 \mathrm{ppm}$ in deep troposphere, an external flux of $1 \times 10^{8} \mathrm{~cm}^{-2} \mathrm{~s}^{-1}$ and the eddy diffusion profile "A" of Romani et al. (1993). Dark blue (label $\mathrm{Pb}$ ): same, but with the eddy diffusion profile "B" of Romani et al. The corresponding $\mathrm{CO}$ and eddy $K$ profiles are shown in the insets. The decrease of $\mathrm{CO}$ at $p<0.001$ mbar is due to molecular diffusion.

is present in the troposphere, since the absorption could conceivably be formed in the lower stratosphere ( 4 mbar $<p<$ 100 mbar) where temperatures are colder than the tropospheric continuum near $230 \mathrm{GHz}(93 \mathrm{~K})$. However, we found models in which $\mathrm{CO}$ is restricted to pressures less than 100 mbar produce too narrow an absorption (Fig. 3). We then tested "two-level" models, in which CO was characterized by a deep value $\left(q_{1}\right)$ below a given pressure $p_{s}$, and a high altitude value $\left(q_{2}\right)$ at pressures less than $p_{s}$. The best fit to the grand average line was achieved for $q_{1}=0.5 \mathrm{ppm}, q_{2}=1.0 \mathrm{ppm}$, and $p_{s}=20 \mathrm{mbar}$ (Fig. 3). The accuracy on these mole fractions is about $15 \%$, and the $p_{s}$ level is determined to within a factor of 2 .

A higher mixing ratio in Neptune's middle and upper stratosphere compared to the troposphere/lower stratosphere value implies the existence of an external source of $\mathrm{CO}$ along with the internal source that is responsible for the deep abundance. The excess of column density that must be maintained by this external source is $2.4 \times 10^{18} \mathrm{~cm}^{-2}$, to within a factor of 2. Physical profiles of $\mathrm{CO}$ were generated by solving the vertical transport equation, accounting for eddy and molecular transport. Because $\mathrm{CO}$ is chemically stable, its downward 
flux $\Phi$ is constant and the CO mole fraction $q(z)$ is given by $K(z) n(z) \frac{\mathrm{d} q(z)}{\mathrm{d} z}=\Phi$ (omitting here molecular transport for simplicity). CO profiles were generated for a series of values of $\Phi$, with the boundary condition $q(z=-\infty)=5 \times 10^{-7}$. Two $K(z)$ profiles were used, namely the "A" and "B" profiles of the Romani et al. (1993) photochemical model. These profiles have minimum values of 600 and $2000 \mathrm{~cm}^{2} \mathrm{~s}^{-1}$, respectively, at the tropopause. Below this level, we assumed that $K(z)$ is proportional to $n(z)$. These physical models match the data for a CO flux of $(1 \pm 0.3) \times 10^{8} \mathrm{~cm}^{-2} \mathrm{~s}^{-1}$. However, CO profiles resulting from the eddy $K$ "A" profile tend to produce somewhat too narrow an emission, and profile "B", characterized by very rapid mixing down to 0.5 mbar, allows a slightly better match to the data. This is consistent with the findings of Romani et al. (1993) who also favored the eddy $K$ profile "B" on the basis of the $\mathrm{C}_{2} \mathrm{H}_{2}$ and $\mathrm{C}_{2} \mathrm{H}_{6}$ abundance.

\section{Discussion and conclusions}

We find a vertically non-uniform $\mathrm{CO}$ profile with an abundance at $p<20$ mbar essentially twice higher than the deep (0.1-2 bar) abundance. While reiterating that our finding is exclusively based on fitting the global $\mathrm{CO}(2-1)$ line shape, we note that our best fit models of Fig. 3 predict a brightness temperature of $74 \mathrm{~K}$ at $230.538 \mathrm{GHz}$ and $500 \mathrm{MHz}$ resolution (vs. $66 \mathrm{~K}$ for uniform $\mathrm{CO}=1 \mathrm{ppm}$ ). This is consistent with the recent Marten et al. (2005) measurements, indicating $T_{B}=73 \pm 4 \mathrm{~K}$ at this frequency.

We find a deep $\mathrm{CO}$ abundance (1.3-2) times lower than the previous estimates of Rosenqvist et al. (1992) and Marten et al. (1993). In the framework of the Lodders \& Fegley (1994) thermochemical equilibrium model, this, however, does not alleviate much the need to invoke a huge oxygen abundance, since the CO mixing ratio at observable levels is an extremely sensitive function of the oxygen abundance, increasing typically by 5 orders of magnitude for a factor-of-10 increase in the oxygen enrichment. Bézard et al. (2002) note, however, that the chemical scheme used by the authors is kinetically too ambitious, which may require a reassessment of their work.

Our primary new result is that of an additional, external source of $\mathrm{CO}$, with a rate of $\sim 1 \times 10^{8} \mathrm{~cm}^{-2} \mathrm{~s}^{-1}$. This is 10-500 times the magnitude of the external flux of $\mathrm{H}_{2} \mathrm{O}$ into Neptune, as estimated by Feuchtgruber et al. (1997) for the Romani et al. (1993) "B" and "A" models respectively. The situation is similar to Jupiter, where - excluding the recent input due to comet Shoemaker Levy 9 - the ratio of the $\mathrm{CO}\left((1.5-10) \times 10^{6} \mathrm{~cm}^{-2} \mathrm{~s}^{-1}\right)$ to $\mathrm{H}_{2} \mathrm{O}\left(<8 \times 10^{4} \mathrm{~cm}^{-2} \mathrm{~s}^{-1}\right)$ deposition rates is larger than 20 and may be as large as 250 (Lellouch et al. 2002; Bézard et al. 2002). Large $\mathrm{CO} / \mathrm{H}_{2} \mathrm{O}$ ratios seem to be inconsistent with an oxygen supply by interplanetary grains. At Jupiter, Bézard et al. (2002) favored the case for a stratospheric $\mathrm{CO}$ resulting from the downward diffusion of material delivered by the impact of (sub)kilometersize comets at sub-millibar levels. We suggest that the same mechanism takes place on Neptune. The timescale for diffusion down to 20 mbar is $\tau \sim 2 H^{2} / K_{20}$ mbar. Taking $H=30 \mathrm{~km}$ and $K_{20 \mathrm{mbar}}=3000 \mathrm{~cm}^{2} \mathrm{~s}^{-1}$, this gives $\tau=190$ years. Integrated over this timescale and the surface of Neptune, the measured flux gives an input of $4.7 \times 10^{37} \mathrm{CO}$ molecules, or $\sim 2.2 \times 10^{15}$ CO grams. This is typically 3 times the SL9 delivery of CO to Jupiter, and may be produced by a $2 \mathrm{~km}$ diameter comet with density 0.5 and $50 \% \mathrm{CO}$ yield at impact. We thus tentatively suggest that the stratospheric $\mathrm{CO}$ we currently observe was produced by a $2 \mathrm{~km}$-comet impact 200 years ago. We note that smaller and more recent impacts could provide the required equivalent flux, but not the presence of external $\mathrm{CO}$ down to the 20-mbar level. Additional aspects supporting this scenario are: (i) the $\mathrm{CO}$ resulting profile after 200 years would mimick the two-level profile of Fig. 3; (ii) the external $\mathrm{CO} / \mathrm{HCN}$ ratio in Neptune's stratosphere above 20 mbar is about 500, but the condensation of HCN near 3 mbar removes $85 \%$ of its column; the corrected ratio is $\sim 75$, similar to what was measured in the SL9 debris, suggesting that the hypothesized event may have produced $\mathrm{HCN}$ as well. Possible problems with the comet scenario, however, are that (i) CS was detected after the SL9 collision but not in Neptune (Moreno 1998), and (ii) collisions of $2 \mathrm{~km}$-comets with Neptune are expected to occur only once every 8000 years according to Bottke et al. (2002), though, based on cratering rates on the Galilean satellites, these estimates might be pessimistic by a factor of 10 (Zahnle et al. 1998).

\section{References}

Bézard, B., Romani, P. N., Conrath, B. J., \& Maguire, W. C. 1991, J. Geophys. Res., 96, 18961

Bézard, B., Romani, P. N., Feuchtgruber, H., \& Encrenaz, T. 1999, ApJ, 515, 868

Bézard, B., Lellouch, E., Strobel, D. F., et al. 2002, Icarus, 159, 95

Bottke, W. F., Jr., Morbidelli, A., Jedicke, R., et al. 2002, Icarus, 156, 399

Coustenis, A., Salama, A., Lellouch, E., et al. 1998, A\&A, 336, L85

Encrenaz, T., Serabyn, E., \& Weisstein, E. W. 1996, Icarus, 124, 616

Encrenaz, T., Lellouch, E., Drossart, P., et al. 2004, A\&A, 413, L5

Feuchtgruber, H., Lellouch, E., de Graauw, T., et al. 1997, Nature, 389, 159

Guilloteau, S., Dutrey, A., Marten, A., \& Gautier, D. 1993, A\&A, 279, 661

Lellouch, E., Bézard, B., Moses, J. I., et al. 2002, Icarus, 159, 112

Lellouch, E. 1995, in The collision of comet Shoemaker-Levy 9 and Jupiter, ed. K. S. Noll, H. A. Weaver, \& P. D. Feldman, STScI Symp. Ser., 213

Lodders, K., \& Fegley, B., Jr. 1994, Icarus, 112, 368

Marten, A., Gautier, D., Owen, T., et al. 1993, ApJ, 406, 285

Marten, A. H. E., Matthews, T., Owen, et al. 2005, A\&A, 429, 1097

Moreno, R. 1998, Ph.D. Thesis, Université Paris VI

Moses, J. I., Lellouch, E., Bézard, B., et al. 2000, Icarus, 145, 166

Naylor, D. A., Davis, G. R., Griffin, M. J., et al. 1994, A\&A, 279, 661

Romani, P. N., Bishop, J., Bézard, B., \& Atreya, S. K. 1993, Icarus, 106,442

Rosenqvist, J., Lellouch, E., Romani, P. N., et al. 1992, ApJ, 392, L99

Zahnle, K., Dones, L., \& Levison, H. F. 1998, Icarus, 136, 202 